\title{
Time Delay Compensation Using Equivalent Elastic Force Feedback
}

\author{
Eiichi Saito* ${ }^{*}$ Student Member, Seiichiro Katsura* Senior Member
}

(Manuscript received Aug. 6, 2014, revised July 13, 2015)

\begin{abstract}
This paper proposes a novel time-delay compensation based on the feedback of an elastic force from a wave system. In the previous research, the authors derived wave representation of a time-delay system and showed that there is equivalence between a resonant system, which is expressed by the wave equation, and a time-delay system: both systems consist of a position-input system and a wave-transmission system. Considering the above equivalence, we can infer that the cause of vibrations in the time-delay system is the robustness of the position-input system against the wave-transmission system, as in the case of the resonant system. To reduce the robustness, the feedback of an equivalent elastic force, which is derived from the wave representation, is introduced according to the concept of a resonant ratio control, which is one of vibration control method on a two-mass resonant system. Because the feedback of the elastic force can reduce the equivalent mass of the position-input system, the vibrations due to the time delay are suppressed. The disturbance-suppression performance of the proposed method is superior to that of a Smith predictor and a communication disturbance observer because the proposed method can maintain the feedback characteristic. The validity of the proposed method is confirmed by simulation and experimental results.
\end{abstract}

Keywords: motion control, time delay compensation, wave equation, resonant system

\section{Introduction}

Control methods of systems with time delay such as teleoperation systems have long been a topic of research ${ }^{(1)-(5)}$. This study considers a system that includes time delay in both feedback and input, which is shown in Fig. 1. In this system, a controller and a plant are separated by time delay such as a communication system. Teleoperation systems often exhibit this type of structure ${ }^{(6)}$ and controlling the plant in this system is difficult because of time delay. Time delay in the feedback loop is known to make the controlled system vibrate or unstable because time delay has a non-minimum phase lag characteristic. Therefore, time-delay compensation is an important issue for teleoperation systems and this paper focuses on the time-delay compensation of the system shown in Fig. 1.

Many time-delay compensation methods have been proposed so far, such as a Smith predictor ${ }^{(7)}$ and a communication disturbance observer ${ }^{(8)(9)}$, and so on ${ }^{(10)}$. The Smith predictor is the most basic method of time-delay compensation and it is widely used. Communication disturbance observerbased methods are superior to the Smith predictor as they do not require the time-delay model or value.

In previous research ${ }^{(11)(12)}$, the authors proposed to suppress vibration in a resonant system by using reflected wave rejection and a wave compensator, based on the communication disturbance observer. In this field, the resonant system (composed of an arbitrarily long series of springs and masses) is modeled with a wave equation, which refers to the distributed parameter model. The equivalent transfer function of the wave equation contains a time delay in the internal

\footnotetext{
* Department of System Design Engineering, Keio University 3-14-1, Hiyoshi, Kohoku-ku, Yokohama 223-8522, Japan
}

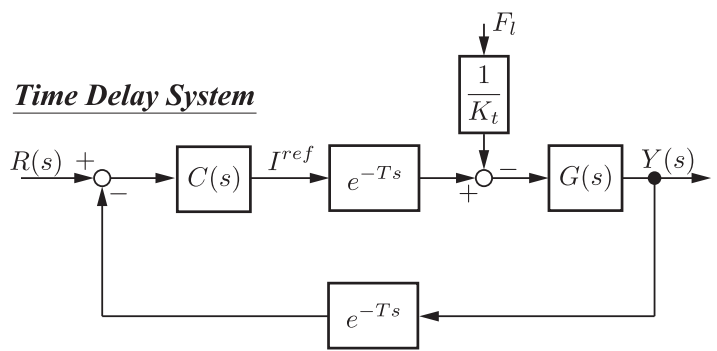

Fig. 1. Block diagram for position control of time-delay system dealt with in this paper

feedback, which suggests that the reflected wave is a cause of vibration. The vibration in the resonant system can be suppressed by using a time-delay compensation method. Considering the above tight connection between time-delay and wave systems, wave representation of a time-delay system was derived, and a reflected wave in the time-delay system was defined $^{(13)}$. In the contribution ${ }^{(13)}$, the remarkable fact is obtained that the wave representation of a time-delay system comprises two parts: a position-input system and a wavetransmission system. The construction is same as that of the resonant system modeled with the wave equation derived in the previous research.

Focusing on the above equivalence of time-delay and resonant systems, this paper proposes a novel time-delay compensation by using equivalent elastic force feedback, which is like vibration control of a two-mass resonant system. In general, the vibration in the resonant system occurs when the motor is robust against the load side of resonant system because the vibration is related to inertia ratio between those of motor and load ${ }^{(14)(15)}$. In particular, if the motor implements a disturbance observer ${ }^{(16)}$, the vibration heavily occurs on the load side of resonant system because equivalent mass of the motor 
is equal to infinity. For instance, a resonant ratio control ${ }^{(17)}$, which is one of vibration control method of a two-mass resonant system, introduces reaction force feedback to reduce the robustness against the load side of resonant system, which contributes to suppress the vibration. Considering the structure of the wave representation of the time-delay system, it is interpreted that vibration or destabilization are caused by the robustness of the position-input system against the wavetransmission system. By using the above concept, the proposed method introduces equivalent elastic force feedback of the time-delay system to reduce the equivalent mass of position-input system in the time-delay system. As a result, the vibration due to time delay is suppressed. Advantage of the proposed method over the Smith predictor and the communication disturbance observer is higher performance of the disturbance suppression because the direct-current component of feedback signals is maintained.

This paper is organized as follows. In Sect. 2, equivalence between time-delay and resonant systems which is derived in the previous research is briefly explained. Section 3 proposes the time-delay compensation based on equivalent elastic force feedback. In Sects. 4 and 5, the validity of the proposed method is confirmed by simulation and experimental results. Finally, this paper is concluded in Sect. 6.

\section{Equivalence of Time-Delay and Resonant Sys- tems ${ }^{(13)}$}

Before explaining the proposed time-delay compensation, an equivalence of time-delay and resonant systems is introduced in the cases that they are modeled with a wave equation.

2.1 Modeling of Resonant System by Using Wave Equation The method previously proposed by the authors ${ }^{(11)}$, which is in the field of the vibration control on a resonant system, considers the distributed position of the resonant system as shown in Fig. 2. Figure 2 can be also understood as an $N$-mass resonant system, when $N$ tends to infinite. In Fig. 2, $u(t), y(t, x)$, and $L$ stand for the position input generated by the actuator, the displacement at $x$ in $t$, and the length of the resonant system, respectively. The control objective of the system is that the position response at $x=L$ corresponds to a position command of the actuator, without residual vibrations. It is assumed that the motor implements the disturbance observer, and the response of motor is not affected by the reaction force from the load side of the resonant system. Hence, the modeling of load side of the resonant system is explained below.

There are many models for the resonant system, such as a two-mass resonant system, a multi-mass resonant system, a

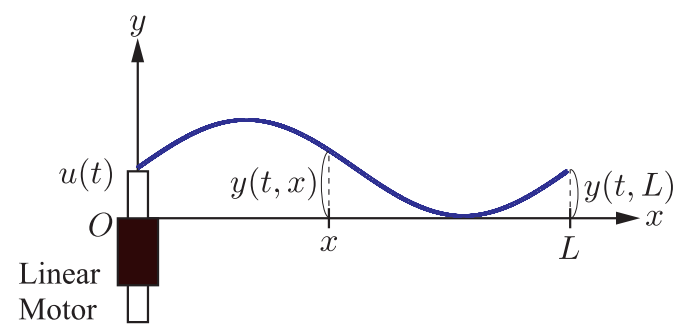

Fig. 2. Wave system wave equation, and so on. In our method, the load side of the resonant system is modeled as a wave equation, which is one of the distributed parameter model that can consider high order resonances. Hence, the motion equation of the resonant system is expressed as

$$
\frac{\partial^{2} y(t, x)}{\partial t^{2}}=c^{2} \frac{\partial^{2} y(t, x)}{\partial x^{2}}
$$

where $c$ stands for the propagation velocity of the wave. The boundary conditions of the resonant system in the Laplace domain are represented as

$$
\begin{aligned}
& Y(s, 0)=U(s) \cdots \cdots \cdots \cdots \cdots \cdots \cdots \cdots \cdots \cdots \\
& \frac{\partial Y(s, L)}{\partial x}=0
\end{aligned}
$$

where $s$ denotes the Laplace operator. Eq. (2) means that position input is applied at $x=0$ by the actuator and (3) means that there is a free end at $x=L$. The position input $U(s)$ means the motor position which is robust against the reaction force from the wave system because the motor implements a disturbance observer ${ }^{(16)}$ and acceleration control is realized. By using the boundary conditions, and the transfer function from the position input $U(s)$ to the position $Y(s, x)$ can be derived $^{(18)}$ as

$$
\frac{Y(s, x)}{U(s)}=\frac{e^{-\frac{x}{c} s}+e^{-\frac{2 L-x}{c} s}}{1+e^{-\frac{2 L s}{c}}} .
$$

To control the position $Y(s, L)$ at the boundary, the transfer function from the position input to the position at $x=L$ is derived as

$$
\frac{Y(s, L)}{U(s)}=\frac{2 e^{-\frac{L s}{c}}}{1+e^{-\frac{2 L s}{c}}}
$$

It is found that the transfer function contains time delays. Additionally, there is a time delay in the denominator of the transfer function. The time delay leads the position $Y(s, L)$ to vibrate. The block diagram of the wave system (5) and motor for generating the position input to wave system is shown in Fig. 3. $R(s), \ddot{X}^{r e f}, K_{p}$ and $K_{d}$ stand for the position command, the acceleration reference, the proportional gain, and differential gain, respectively. Figure 3 shows that there is a wave reflected at the boundary at $x=L$ and the reflected wave travels to the position at $x=0$. The negative feedback represents the reflected wave which, in turn, causes the vibration.

2.2 Modeling of Time-Delay System by Using Wave Equation This subsection explains the modeling of the time-delay system and introduces the wave representation of this system. In addition, the equivalence between the timedelay and resonant systems is described.

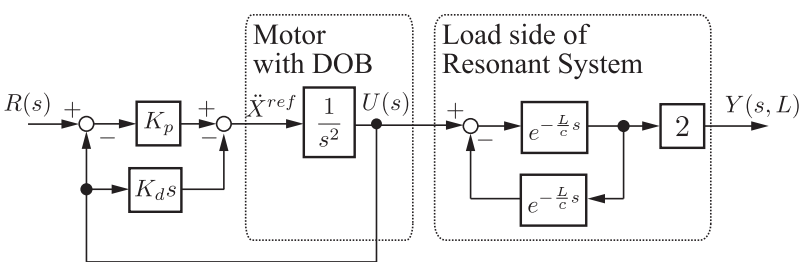

Fig. 3. Block diagram of resonant system modeled as wave system 
2.2.1 Time-Delay System Firstly, the positionfeedback system including time delay is explained. The block diagram of the time-delay system is shown in Fig. 1. $T, C(s), G(s), I^{r e f}, Y(s), F_{l}$, and $K_{t}$ denote the input/output time-delay, the position controller, the plant, the current reference and the output (i.e., position), the external load force, and the torque/thrust-force coefficient, respectively. Here, the plant $G(s)$ is represented as a motor, and the controller $C(s)$ is represented as a PD controller:

$$
\begin{aligned}
G(s) & =\frac{K_{t}}{M s^{2}} \cdots \cdots \cdots \\
C(s) & =\frac{M_{n}}{K_{t n}}\left(K_{p}+K_{d} s\right)
\end{aligned}
$$

where $M$ stands for the inertia/mass. Subscript ${ }_{n}$ denotes the nominal value. It is noted that the term $M_{n} / K_{\text {tn }}$ in (7) is for canceling out the term $K_{t} / M$ in (6). The transfer functions from the position command $R(s)$ and the load force $F_{l}$ to the position $Y(s)$ is represented as

$$
\begin{aligned}
\frac{Y(s)}{R(s)} & =\frac{C(s) G(s) e^{-T s}}{1+C(s) G(s) e^{-2 T s}} \cdots \ldots \ldots \ldots \ldots \ldots \ldots \ldots \ldots \ldots \ldots
\end{aligned}
$$

From (8) and (9), there is a time delay in the denominator of the transfer function. The time delay has possibility which makes the system unstable because the phase margin is reduced by the phase lag characteristic of time delay. Therefore, the time delay needs to be compensated for in order to stabilize the controlled system. In this paper, the time delay is compensated for by the concept of elastic force feedback which is described in the detail in Sect. 3.

2.2.2 Wave Representation of Time-Delay System

This part describes the wave representation of the timedelay system shown in Fig. 1. For simplicity, $F_{l}=0$ is assumed in this part. Firstly, a distributed position $\tilde{y}(t, x)$ $(0<x<\tilde{L})$ is introduced. $\tilde{L}$ is the equivalent system length of the time-delay system, and the position $Y(s)$ is equal to the position $\tilde{Y}(s, L)$. The distributed position $\tilde{y}(t, x)$ satisfies the wave equation:

$$
\frac{\partial^{2} \tilde{y}(t, x)}{\partial t^{2}}=\tilde{c}^{2} \frac{\partial^{2} \tilde{y}(t, x)}{\partial x^{2}}
$$

where $\tilde{c}$ stands for the equivalent propagation velocity of the wave in the time-delay system. It is assumed that the time delay $T$ satisfies $T=\tilde{L} / \tilde{c}$ where $\tilde{L}$ denotes the equivalent system length. The boundary conditions of the time-delay system in the Laplace domain are defined as

$$
\begin{aligned}
\tilde{Y}(s, 0) & =\frac{C(s) G(s)}{1+C(s) G(s)} R(s) \cdots \ldots \\
\tilde{Y}(s, \tilde{L}) & =Y(s) \\
& =\frac{C(s) G(s) e^{-\frac{\tilde{L}}{\tilde{c}} s}}{1+C(s) G(s) e^{-2 \frac{\tilde{L}}{\tilde{c}}}} R(s) .
\end{aligned}
$$

Equation (11) means that the position input for the timedelay system is represented by using the wave equation. On the other hand, (12) is set as the position response $Y(s)$ and is rewritten by using the transfer function (8). It is noted

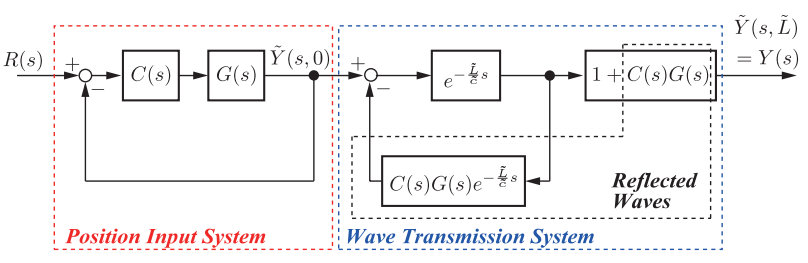

Fig. 4. Block diagram of wave representation of timedelay system from $R(s)$ to $\tilde{Y}(s, L)$

that, this paper assumes the input time-delay $T_{i}$ equals to output time delay $T_{o}$ (i.e. $T_{i}=T_{o}=T$ ). If there exists the difference between them, (12) should be modified as $\tilde{Y}(s, \tilde{L})=Y(s) e^{\left(\left(T_{i}+T_{o}\right) / 2-T_{i}\right) s}$ because the position $Y(s)$ is not located at the end of the wave system $\tilde{L}$.

As well as way of the case in the resonant-system, the transfer function from $\tilde{Y}(s, 0)$ to $\tilde{Y}(s, x)$ can be derived as

$$
\tilde{Y}(s, x)=\frac{e^{-\frac{x}{\tilde{c}} s}+C(s) G(s) e^{-\frac{2 \tilde{L}-x}{\tilde{c}} s}}{1+C(s) G(s) e^{-2 \frac{\tilde{L}}{\tilde{c}} s}} \tilde{Y}(s, 0) . \cdots \cdots \cdots
$$

Finally, by substituting $x=\tilde{L}$ to (13), the transfer function of the wave representation in the time-delay system is derived as

$$
\tilde{Y}(s, \tilde{L})=\frac{e^{-\frac{\tilde{L}}{\tilde{c}} s}(1+C(s) G(s))}{1+C(s) G(s) e^{-2 \frac{\tilde{L}}{\tilde{c}} s}} \tilde{Y}(s, 0) . \cdots \cdots \cdots
$$

It is noted that the transfer function from $R(s)$ to $\tilde{Y}(s, 0)$ is represented as (11). Hence, the block diagram of timedelay system in Fig. 1 can be transformed into an equivalent block diagram based on the wave equation in Fig. 4. Figure 4 shows the block diagram for the wave representation of timedelay system. The wave representation of the time-delay system also comprises a position-input system and a wavetransmission system. In other words, this structure implies that the position-input system is not affected by the wavetransmission system which is the same situation as the resonant system.

\section{Time-Delay Compensation Based on Feedback of Elastic Force from Wave System}

3.1 Feedback of Equivalent Elastic Force According to the wave representation of the time-delay system shown in Fig. 4, it is interpreted that vibration or destabilization are caused by the robustness of the position-input system against the wave-transmission system. This aspect comes from vibration control of the resonant system. In general, the load position of a two-mass resonant system becomes vibrating when the mass of the motor is greater than the mass of the load $^{(15)}$. In particular, if the motor implements a disturbance observer, the vibration becomes heavy because the equivalent mass against the reaction force becomes infinity. Resonant ratio control ${ }^{(17)}$, which is a vibration control method, introduces reaction force feedback to reduce the equivalent mass of the motor against the reaction force from the resonant system. As a result, the vibration is suppressed. Here, this concept of resonant ratio control is applied to the timedelay system.

Firstly, the deformation of the wave system is derived to define the elastic force in time-delay system. The deformation is represented as the partial differentiation of the position 


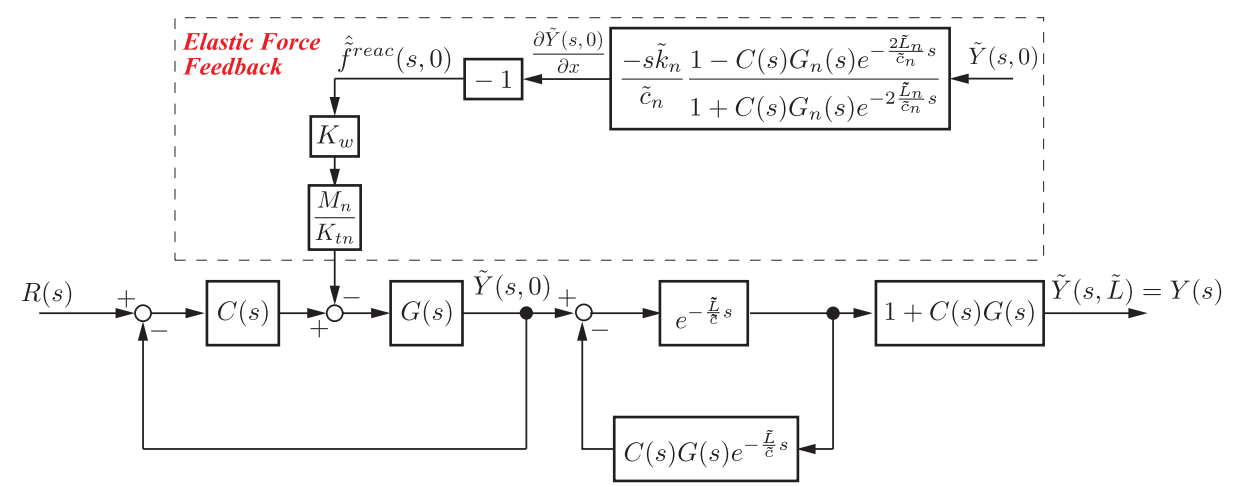

Fig. 5. Block diagram of elastic force feedback in time-delay system expressed as wave

$\tilde{Y}(s, x)$ with respect to $x$ :

$$
\frac{\partial \tilde{Y}(s, x)}{\partial x}=\frac{-s}{\tilde{c}} \frac{e^{-\frac{x}{\tilde{c}} s}-C(s) G(s) e^{-\frac{2 \tilde{L}-x}{\tilde{c}} s}}{1+C(s) G(s) e^{-2 \frac{\tilde{L}}{\tilde{c}} s}} \tilde{Y}(s, 0) . \cdots
$$

By using the above equation and the equivalent stiffness $\tilde{k}$, the elastic force at $x$ is derived as

$$
\tilde{f}^{r e a c}(s, x)=-\tilde{k} \frac{\partial \tilde{Y}(s, x)}{\partial x} .
$$

It is noted that the reason why "-1" exists in right hand of (16) is that the definition of sign of torsion in the wave equation, which is $\lim _{\Delta x \rightarrow 0}(Y(s, x+\Delta x)-Y(s, x)) / \Delta x$, is opposite to the definition of two-mass resonant system, which is motor position minus the load position. Because the position-input system is located at $x=0$, the elastic force acting on the position-input system is derived as

$$
\begin{aligned}
\tilde{f}^{r e a c}(s, 0) & =-\tilde{k} \frac{\partial \tilde{Y}(s, 0)}{\partial x} \\
& =\frac{s \tilde{k}}{\tilde{c}} \frac{1-C(s) G(s) e^{-\frac{2 \tilde{L}}{\tilde{c}} s}}{1+C(s) G(s) e^{-2 \frac{\tilde{L}}{\tilde{c}} s}} \tilde{Y}(s, 0) . \cdots \cdots
\end{aligned}
$$

The concept diagram of the elastic force feedback for vibration suppression on time-delay system is shown in Fig. 5. In Fig. 5, $K_{w}$ denotes the elastic force gain. By the feedback of the equivalent elastic force, motion equation of the position-input system is changed as

$$
s^{2} \tilde{Y}(s, 0)=\frac{K_{t}}{M}\left[C(s)(R(s)-\tilde{Y}(s, 0))-\frac{M_{n}}{K_{t n}} K_{w} \hat{\tilde{f}}^{r e a c}(s, 0)\right]
$$

where $\hat{\tilde{f}}^{\text {reac }}(s, 0)$ denotes an estimated equivalent elastic force. Therefore, an equivalent mass $\tilde{M}^{e q}$ in Fig. 5, which means the influence on the acceleration which is given by elastic force $\hat{\tilde{f}}^{r e a c}(s, 0)$, is represented as

$$
\tilde{M}^{e q} \approx \frac{1}{K_{w}}
$$

In (19), it is assumed that the nominal values $\left(K_{t n}\right.$ and $\left.M_{n}\right)$ are equal to the actual values $\left(K_{t}\right.$ and $\left.M\right)$. It can be seen that the equivalent mass equals to infinity when there is no feedback of the elastic force $\left(K_{w}=0\right)$. In this case, the position-input system is robust against wave-transmission system, which results to induce the vibration. It is also found that the elastic force feedback can reduce the equivalent mass of the input system. Therefore, it is expected to suppress the vibration due to the time delay because robustness of the input system against elastic force is reduced.

There is a problem with implementing the control system shown in Fig. 5: It is needed to know the equivalent velocity of the wave $\tilde{c}$ and stiffness $\tilde{k}$. Here, the elastic force gain is redefined as

$$
K_{w}^{\prime}=K_{w} \frac{\tilde{k}}{\tilde{c}} \cdot
$$

The gain is determined by cut and try in this paper. By using the above redefined elastic force gain, the reaction force feedback can be conducted as

$$
\begin{aligned}
& K_{w} \hat{\tilde{f}}^{r e a c}(s, 0)=K_{w} \frac{s \tilde{k}_{n}}{\tilde{c}_{n}} \times \\
& \frac{1-C(s) G_{n}(s) e^{-\frac{2 \tilde{L}_{n}}{\tilde{c}_{n}} s}}{1+C(s) G_{n}(s) e^{-2 \frac{\tilde{L}_{n}}{\tilde{c}_{n}} s}} \tilde{Y}(s, 0) \\
& =K_{w}^{\prime} s \times \\
& \frac{1-C(s) G_{n}(s) e^{-\frac{2 \tilde{L}_{n}}{\tilde{c}_{n}} s}}{1+C(s) G_{n}(s) e^{-2 \frac{\tilde{L}_{n}}{\tilde{c}_{n}} s}} \tilde{Y}(s, 0) . \cdots
\end{aligned}
$$

By using (11) and (12), Eq. (21) can be rewritten as

$$
\begin{aligned}
K_{w} \hat{\tilde{f}}^{r e a c}(s, 0)= & K_{w}^{\prime} s \frac{C(s) G_{n}(s)}{1+C(s) G_{n}(s)} \times \\
& \left(R^{\prime}(s)-2 e^{-T s} Y(s)\right) .
\end{aligned}
$$

where $R^{\prime}(s)$ contains the reference signal $R(s)$ and the compensation based on elastic force feedback. Here, a delay model is not required for the implementation of the elastic force feedback since the delayed output, i.e. $e^{-T s} Y(s)$, is already provided to the controller. The block diagram of timedelay compensation based on elastic force feedback for actual implementation is shown in Fig. 6. In Fig. 6, $Y^{c m p}$ means the compensation value including the equivalent elastic force. The compensation value $Y^{c m p}$ for time delay is calculated as

$$
Y^{c m p}=\frac{1}{C(s)} \frac{M_{n}}{K_{t n}} K_{w} \hat{\tilde{f}}^{r e a c}(s, 0) .
$$

Feedback of $Y^{c m p}$ to the position command makes a same effect as shown in Fig. 5. The current reference which is transmitted to plant side is represented as

$$
\ddot{X}^{r e f}=C(s)\left(\frac{C(0)}{C(s)} R(s)-Y^{c m p}-e^{-T s} Y(s)\right) \ldots \ldots
$$




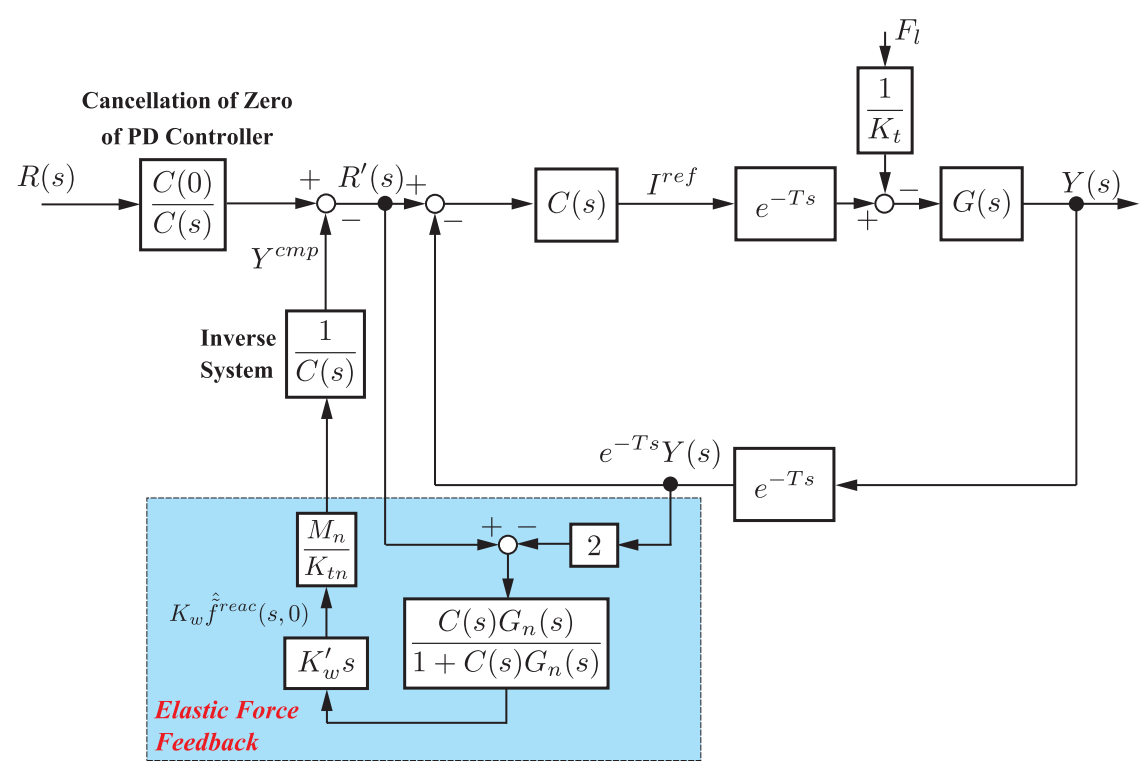

Fig. 6. Equivalent block diagram of Fig. 5 for implementation (the proposed method)

It is noted that, in this paper, the zero of PD controller is canceled in order to improve the performance of the vibration suppression; it has the same effect as a $\mathrm{P}$ controller with velocity minor loop. In addition, the control gains $\left(K_{p}, K_{d}, K_{w}^{\prime}\right)$ in the proposed method have clear physical meanings. Therefore, it is expected to set those gains easily by cut and try.

3.2 Disturbance Suppression Performance of Proposed Method In contrast to the well-known Smith predictor and communication disturbance observer (CDOB), the proposed method maintains the feedback characteristics. The transfer function from the disturbance $F_{l}$ to position response $Y(s)$ is represented as

$$
G_{F Y}=\frac{-1}{M} \frac{s^{2}+\left(K_{d}+K_{w}^{\prime}\right) s+K_{p}}{\operatorname{Den}(s)} .
$$

where

$$
\begin{aligned}
\operatorname{Den}(s)= & s^{4}+\left(K_{d}+K_{w}\right) s^{3}+K_{p} s^{2}+ \\
& {\left[K_{d} s^{3}+\left(K_{p}+K_{d}^{2}-K_{d} K_{w}\right) s^{2}+\right.} \\
& \left.\left(2 K_{p} K_{d}-K_{p} K_{w}\right) s+K_{p}^{2}\right] \alpha e^{-2 T s}, \ldots \ldots \\
\alpha= & \frac{K_{t} M_{n}}{K_{t n} M} . \cdots \ldots \ldots \ldots \ldots \ldots \ldots \ldots \ldots
\end{aligned}
$$

From (25), it turns out that the direct-current (DC) gain of (25) equals to $1 /\left(\alpha M K_{p}\right)$, which means that it is same disturbance suppression performance as a typical PD controller. In other words, constant steady state error occurs as well as that of a typical second-order system with PD controller when a step disturbance acts on the system. On the other hand, with the CDOB and Smith predictor, instead of the control design that does not consider time delay, the feedback characteristic is removed or reduced. In other words, the DC component is removed or reduced from feedback signals by the time-delay compensation. Thus, these methods are easily affected by the disturbance such as a friction, external forces. In contrast, the proposed method does not remove the DC component of the feedback signals. Figure 7 shows the bode diagram of each method regarding the closed-loop transfer function from disturbance to position. From the bode diagram, DC gains of

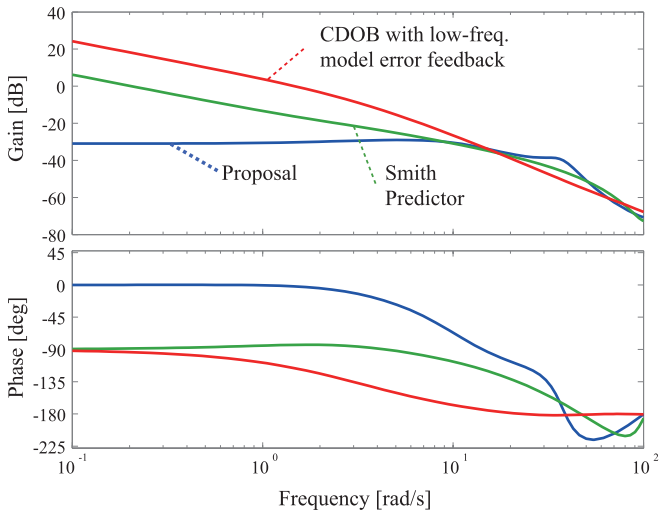

Fig. 7. Bode diagram of the closed-loop transfer function from disturbance to position

the Smith predictor and the CDOB with low-frequency model error feedback ${ }^{(19)}$ are infinite with $20 \mathrm{~dB} / \mathrm{dec}$ (one pole is located at the imaginary axis), which means that steady state error does not occur by the impulse disturbance. On the other hand, DC gain of the proposed method equals to finite value, which means that steady state error does not occur by the impulse disturbance. It is noted that if some kinds of the disturbance observer can be implemented, steady state error does not occur by the step disturbance. Therefore, the disturbance response becomes better than that of CDOB and Smith predictor and the response at steady state is improved.

\section{Numerical Results}

To confirm the effectiveness of the proposed method, simulations of position control are conducted. Simulation parameters are shown in Table 1 . It is assumed that there are no parameter variations in $K_{t}$ and $M$ in the simulations. Additionally, it is also assumed that the bandwidth of current control is enough wide, and it can be neglected. In all the simulations, step input $(R(s)=5.0 \mathrm{~mm})$ is applied.

Firstly, effect of elastic force feedback is confirmed. Figure 8 shows the simulation results when $K_{p}=144, K_{d}=48$, and $K_{w}$ is varied from 0.0 to 24.0 with the step of 4.0 . In this 
Table 1. Simulation parameters

\begin{tabular}{c|l|c}
\hline Parameter & Description & Value \\
\hline \hline$T_{s}$ & Sampling time & $0.2 \mathrm{~ms}$ \\
$K_{t}=K_{t n}$ & $\begin{array}{l}\text { Actual and nominal } \\
\text { thrust-force coefficients }\end{array}$ & $2.7 / 0.81 \mathrm{~N} / \mathrm{A}$ \\
$M=M_{n}$ & Actual and nominal mass of motor & $0.245 \mathrm{~kg}$ \\
\hline
\end{tabular}

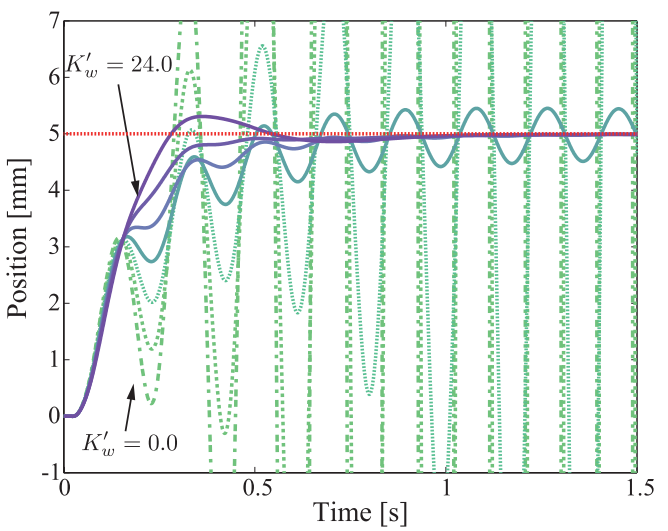

Fig. 8. Simulation results when $K_{w}^{\prime}$ changes from 0.0 to $24.0\left(2 T=50 \mathrm{~ms}, K_{p}=144, K_{d}=48\right)$

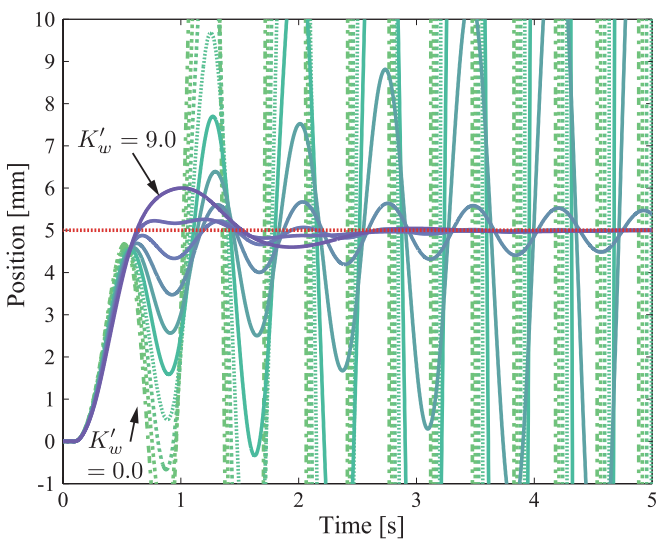

Fig. 9. Simulation results when $K_{w}^{\prime}$ changes from 0.0 to $9.0\left(2 T=200.0 \mathrm{~ms}, K_{p}=16, K_{d}=16\right)$

simulation, the round-trip time delay $2 T$ is equal to $50 \mathrm{~ms}$. When $K_{w}^{\prime}=0$, the response of the motor position is unstable. On the other hand, when $K_{w}^{\prime}$ is gradually increased, the control system is stabilized and the vibration is gradually decreased. The equivalent elastic force feedback is confirmed to be effective at suppressing the vibration due to time delay. However, too much increasing $K_{w}^{\prime}$ makes the system unstable again. Hence, adjustment of the appropriate value of $K_{w}^{\prime}$ is needed.

Figure 9 shows the simulation results in the case of large round-trip time delay $(2 T=200.0 \mathrm{~ms})$. In this simulation, $K_{p}=16, K_{d}=16$, and $K_{w}^{\prime}$ is varied from 0.0 to 9.0 with the step of 1.5. As well as Fig. 8, the feedback of elastic force can stabilize the system. Figure 10 shows the simulation results in the case of $2 T=200.0 \mathrm{~ms}$ with large controller gains $\left(K_{p}=25, K_{d}=20\right)$. From Fig. 10, the feedback of elastic force can stabilize the system. In the case of Fig. 10, $K_{w}^{\prime}$ is varied from 9.0 to 12.0 with the step of 0.5 . However, vibration still remains on the response with optimal gain. Therefore, stability region by using the elastic force feedback

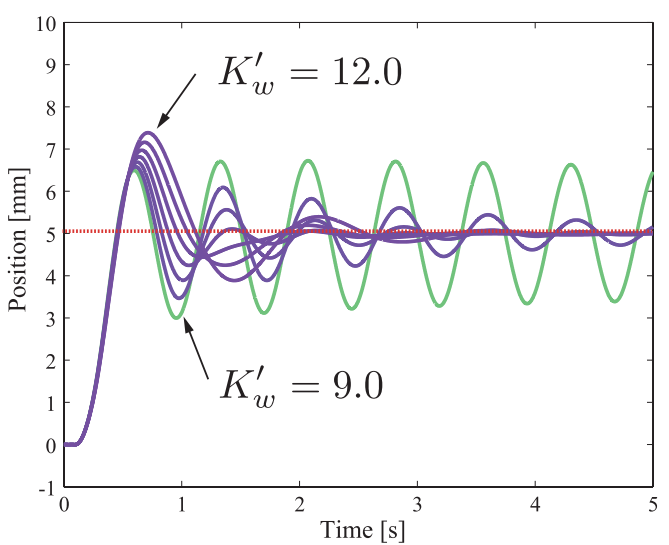

Fig. 10. Simulation results when $K_{w}^{\prime}$ changes from 9.0 to $12.0\left(2 T=200.0 \mathrm{~ms}, K_{p}=25, K_{d}=20\right)$

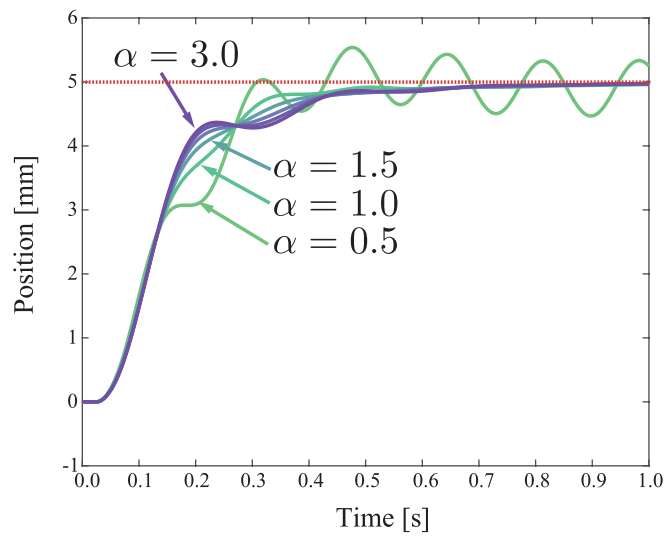

Fig. 11. Simulation results when parameter variations $\alpha$ change from 0.5 to $3.0\left(2 T=50.0 \mathrm{~ms}, K_{p}=144\right.$, $K_{d}=48, K_{w}^{\prime}=20$ )

depends on the value of gains in the position controller.

Figures 9 and 10 imply that the proposed method has weak points which is the variation of time delay (i.e. jitter). From the point of view of robustness against the jitter, performance of the proposed method is inferior to performance of the smith predictor and communication disturbance observer based methods. Therefore, if there exists large variation of time delay, it is needed to use some kinds of jitter compensator (e.g. jitter buffer) for implementation of the proposed method.

In addition, the parameter variation in $K_{t}$ and $M$ also degrades both the control performance and the stability because the elastic force feedback gain is equivalently changed. Figure 11 shows simulation results when parameter variation $\alpha$, which is represented as (27), changes from 0.5 to 3.0 $\left(2 T=50.0 \mathrm{~ms}, K_{p}=148, K_{d}=48, K_{w}^{\prime}=20\right)$. Interpreting the results shown in Fig. 11 roughly, if $\alpha$ decreases, the damping ratio provided by the PD controller also decreases. On the other hand, if $\alpha$ increases, the damping ratio increases and the system becomes stable. However, if $\alpha$ increases too much, the system becomes unstable because increasing $K_{p}$ and $K_{w}^{\prime}$ destabilizes the system. If there exists large variation in $K_{t}$ and $M$, a compensator should be designed to maintain robust stability. 


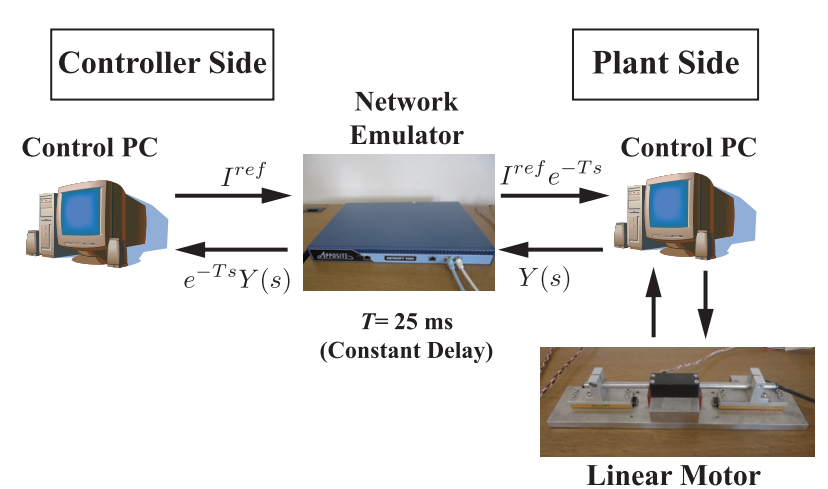

Fig. 12. Experimental setup

\section{Experiments}

5.1 Experimental Setup In order to verify the effectiveness of the proposed method, the position control experiments are performed. The schematic diagram of experimental system is shown in Fig. 12. A linear motor with a optical encoder (resolution capability: $100 \mathrm{~nm}$ ) is used as the controlled plant. A constant time delay is artificially generated by a network emulator. Jitter of the communication delay is not considered. Communication system is realized by using the User Datagram Protocol (UDP), and the sending period is the same as the control period. Real-time control system is realized using Real Time Application Interface 3.7 (RTAI 3.7) on Linux and the control program is written in $\mathrm{C}$ language.

The performance of the proposed method is compared with that of the typical Smith predictor and a communication disturbance observer with low-frequency model error feedback $^{(19)}$ when a step position command is applied. The disturbance estimation observer ${ }^{(20)}$, which is a disturbance observer for time-delay system, is implemented in all methods for reducing the effect of the disturbance such as static friction. The compensation current generated by the disturbance estimated observer is represented as

$$
\begin{aligned}
I_{d i s}^{c m p}= & \frac{g_{d i s}^{2}}{s^{2}+2 g_{d i s} s+g_{d i s}^{2}} \times \\
& {\left[K_{t n} I^{r e f} e^{-T s}-M_{n} s^{2} Y(s) e^{-T s}\right] . }
\end{aligned}
$$

The experimental parameters are listed in Table 2. In this paper, the derivation in the PD controller is implemented by using the pseudo derivation with cut-off frequency $g_{p d}$. The parameters of the compared methods are adjusted to coincide with the time constant of the proposed method. As a result, the parameters of the compared methods are obtained by the cut and try as shown in Table 3.

5.2 Experimental Results

Figures 13-15 compare the experimental results of the three methods. Figure 13 shows that the communication disturbance observer stabilizes the control system. However, the communication disturbance observer based method is not able to eliminate the steady state even if low-frequency model error feedback is implemented. Low-frequency model error feedback is effective for suppressing the modeling error, but is not effective for eliminating the disturbance such as static friction. Figure 14 shows that Smith predictor also stabilizes the control system.
Table 2. Experimental parameters

\begin{tabular}{c|l|c}
\hline Parameter & Description & Value \\
\hline \hline$T_{s}$ & Sampling time & $0.2 \mathrm{~ms}$ \\
$K_{t n}$ & Thrust force coefficient & $2.7 / 0.81 \mathrm{~N} / \mathrm{A}$ \\
$M_{n}$ & Mass of motor & $0.245 \mathrm{~kg}$ \\
$K_{p}$ & Proportional gain & 144 \\
$K_{d}$ & Differential gain & 48 \\
$K_{w}^{\prime}$ & Elastic force gain & 21.0 \\
$g_{p d}$ & Cut-off frequency of & $2000 \mathrm{rad} / \mathrm{s}$ \\
& pseudo derivation & \\
$g_{d i s}$ & Cut-off frequency of & $250 \mathrm{rad} / \mathrm{s}$ \\
& disturbance estimation observer & \\
$2 T=\frac{2 \tilde{L}}{\tilde{c}}$ & Time delay & $50 \mathrm{~ms}$ \\
\hline
\end{tabular}

Table 3. Experimental parameters for CDOB and Smith predictor

\begin{tabular}{c|l|c}
\hline Parameter & Description & Value \\
\hline \hline$K_{p}$ & Proportional gain in Smith Predictor & 324 \\
$K_{d}$ & Differential gain in Smith Predictor & 36 \\
$K_{p}$ & Proportional gain in CDOB & 225 \\
$K_{d}$ & Differential gain in CDOB & 60 \\
$g_{c}$ & Cut-off freq. of CDOB & 1000.0 \\
$g_{e}$ & Cut-off freq. of low-freq. & 2.5 \\
& model error feedback & \\
$g_{p d}$ & Cut-off frequency of & $2000 \mathrm{rad} / \mathrm{s}$ \\
& pseudo derivation & $250 \mathrm{rad} / \mathrm{s}$ \\
$g_{d i s}$ & Cut-off frequency of & \\
& disturbance estimation observer & \\
\hline
\end{tabular}

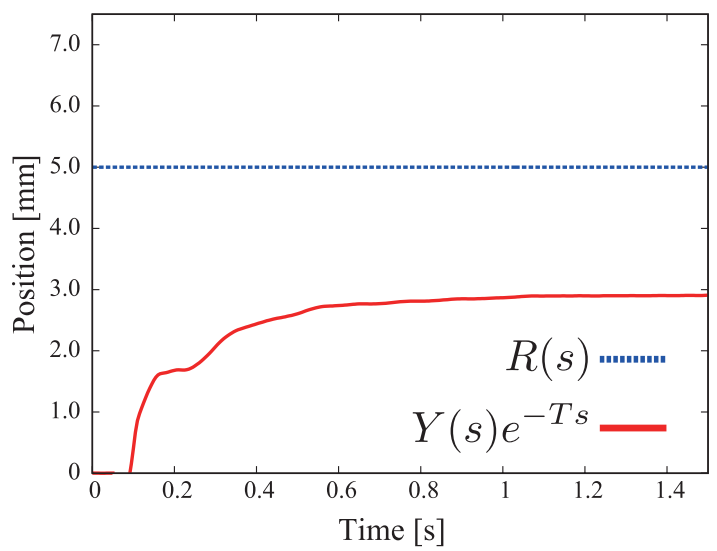

Fig. 13. Experimental result of communication disturbance observer with low-frequency model error feedback

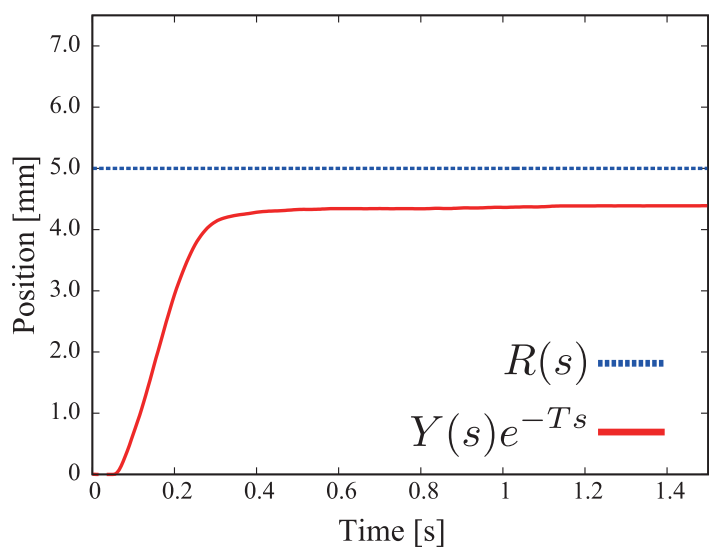

Fig. 14. Experimental result of Smith predictor

However, steady state error is observed due to the disturbance. It is because Smith predictor based method also loses the feedback characteristic as same as the communication 


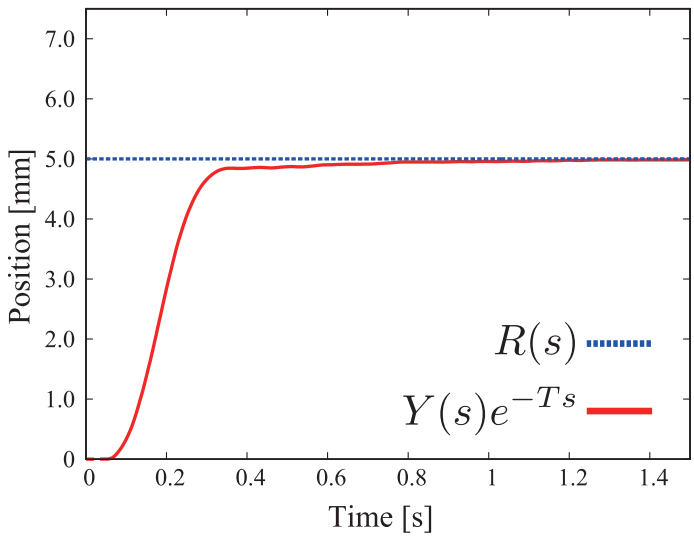

Fig. 15. Experimental result of the proposed method

disturbance observer based method. On the other hand, Figure 15 shows the proposed method can also stabilize the control system. In addition, it is found that the proposed method can eliminate the steady state error because feedback characteristic is maintained. Validity of the proposed method is confirmed by these experimental results.

\section{Conclusions}

This paper proposed the time-delay compensation of a system by using feedback of the equivalent elastic force. The equivalent elastic force was derived by the wave representation of a time-delay system which was proposed in previous research. The wave representation were composed of two parts: a position-input system and a wave-transmission system. The wave representation of a time-delay system had same structure as a resonant system represented by the wave equation. Thus, it could be interpreted that the vibration or destabilization was caused by robustness of the motor against the response of the load side just like a resonant system. According to the physical interpretation, feedback of the elastic force from the wave system was introduced in order to suppress the vibration due to time delay, which is like a resonant ratio control.

Validity of the proposed time-delay compensation was verified by the simulation and experimental results. As the future work, the application of the proposed method to multi-degree of freedom system as well as derivation of the optimal gain $K_{w}^{\prime}$ should be considered. In addition, the variation of the time delay and plant parameters should be taken into account.

\section{Acknowledgment}

This work was partially supported by JSPS KAKENHI Grant Number $15 \mathrm{H} 02236$.

\section{References}

( 1 ) J.P. Richard: "Time-delay Systems: an Overview of Some Recent Advances and Open Problems", Automatica, Vol.39, No.10, pp.1667-1694 (2003)

( 2 ) H. Morioka, A. Sabanovic, A. Uchibori, K. Wada, and M. Oka: "Application of Time-delay-control in Variable Structure Motion Control Systems", The IEEE International Symposium on Industrial Electronics Society, ISIE'05PUSAN, pp.1313-1318 (2001)

( 3 ) K. Gu and S-I. Niculescu: "Survey on Recent Results in the Stability and Control of Time-Delay Systems", ASME Journal of Dynamic Systems, Measurement, and Control, Vol.125, No.2, pp.158-165 (2003)

( 4 ) M. Bowthorpe: "Smith Predictor-Based Robot Control for UltrasoundGuided Teleoperated Beating-Heart Surgery", IEEE Journal of biomedical and Health Informatics, Vol.18, No.1, pp.157-166 (2014)
( 5 ) R. Yang, G. Liu, P. Shi, C. Thomas, and M.V. Basin: "Predictive Output Feedback Control for Networked Control Systems", IEEE Trans. on Industrial Electronics, Vol.61, No.1, pp.512-520 (2014)

( 6 ) A. Suzuki and K. Ohnishi: "Frequency-Domain Damping Design for TimeDelayed Bilateral Teleoperation System Based on Modal Space Analysis", IEEE Trans. on Industrial Electronics, Vol.60, No.1, pp.177-190 (2013)

( 7 ) O.J.M. Smith: "A Controller to Overcome Dead Time", The International Society of Automation Journal, Vol.6, No.2, pp.28-33 (1959)

( 8 ) K. Natori and K. Ohnishi: "A Design Method of Communication Disturbance Observer for Time-Delay Compensation, Taking the Dynamic Property of Network Disturbance Into Account", IEEE Trans. on Industrial Electronics, Vol.55, No.5, pp.2152-2168 (2008)

( 9 ) K. Natori, R. Oboe, and K. Ohnishi: "Stability Analysis and Practical Design Procedure of Time Delayed Control Systems With Communication Disturbance Observer", IEEE Trans. on Industrial Informatics, Vol.4, No.3, pp.185-197 (2008)

(10) A. Denasi, D. Kostic, and H. Nijmeijer: "Time Delay Compensation in Bilateral Teleoperations Using IMPACT", IEEE Trans. on Control Systems Technnology, Vol.21, No.3, pp.704-715 (2013)

(11) E. Saito and S. Katsura: "Vibration Suppression of Resonant System by Using Wave Compensator", The 37th Annual Conference of the IEEE Industrial Electronics Society, IECON'11-MELBOURNE, pp.4105-4110 (2011)

(12) E. Saito and S. Katsura: "Position Control of a Resonant System with Suppression of Load Force Using Wave Observer", IEEJ Journal of Industry Applications, Vol.3-D, No.1, pp.18-25 (2014)

(13) E. Saito, R. Oboe, and S. Katsura: "Time Delay Compensation Method Based on Reflected Wave Rejection", The 39th Annual Conference of the IEEE Industrial Electronics Society, IECON'13-VIENNA (2013)

(14) Y. Hori, H. Sawada, and C. Yeonghan: "Slow Resonance Ratio Control for Vibration Suppression and Disturbance Rejection in Torsional System", IEEE Trans. on Industrial Electronics, Vol.46, No.1, pp.162-168 (1999)

(15) N. Takesue and J. Furusho: "A Consideration on Inertia-Ratio and Damping Property of Vibration of Two-Inertia System", T. IEE Japan, Vol.121-D, No.2, pp.283-284 (2001)

(16) K. Ohnishi, M. Shibata, and T. Murakami: "Motion Control for Advanced Mechatronics", IEEE/ASME Trans. on Mechatronics, Vol.1, No.1, pp.56-67 (1996)

(17) K. Yuki, T. Murakami, and K. Ohnishi: "Vibration Control of 2 mass Resonat System by Resonant Ratio Control", Proc. of the 1993 IEEE International Conference on Industrial Electronics, Control, and Instrumentation, IECON'93-MAUI, Vol.3, pp.2009-2014 (1993)

(18) E. Kreyszig: "Advanced Engineering Mathematics", Wiley (2005)

(19) A. Suzuki and K. Ohnishi: "Improvement in Steady-State Accuracy of TimeDelayed Control Systems with Communication Disturbance Observer by Low-Frequency Model Error Feedback", The IEEJ Trans. on Industry Applications, Vol.133-D, No.9, pp.861-867 (2013)

(20) T. Takehara, T. Kunitake, H. Hashimoto, and F. Harashima: "The Control for the Disturbance in the System with Time Delay", The 4th International Workshop on Advanced Motion Control, AMC'96-MIE, Vol.1, pp.349-353 (1996)

Eiichi Saito (Student Member) received his B.E. degree in system de-

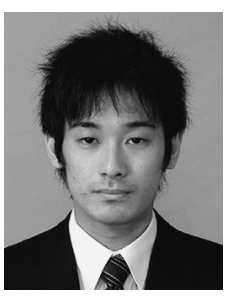
sign engineering and his M.E. degrees in integrated design engineering from Keio University, Yokohama, Japan, in 2011 and 2012. He is currently working toward the Ph.D. degree at Keio University. His research interests include vibration control, haptics, and time-delay compensation. Mr. Saito received the IEEE Industrial Electronics Society Best Conference Paper Award in 2012.

Seiichiro Katsura (Senior Member) received the B.E. degree in sys-

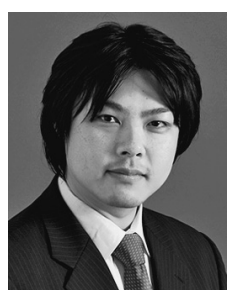
tem design engineering and the M.E. and Ph.D. degrees in integrated design engineering from Keio University, Yokohama, Japan, in 2001, 2002 and 2004, respectively. From 2003 to 2005, he was a Research Fellow of the Japan Society for the Promotion of Science. From 2005 to 2008, he worked at Nagaoka University of Technology, Nagaoka, Niigata, Japan. Since 2008, he has been with Keio University, Yokohama, Japan. His research interests include human support, wave system, systems energy conversion, and industrial electronics. Prof. Katsura received the IEEE Industrial Electronics Society Best Conference Paper Award in 2012. 